\title{
Personal Impressions from IAU Symposium 192: The Stellar Content of the Local Group
}

\author{
J.E. Hesser \\ National Research Council of Canada, Herzberg Institute of \\ Astrophysics, Dominion Astrophysical Observatory, Victoria, BCV8X \\ 4M6, Canada
}

\section{Introduction}

This has been an extremely rich, rewarding meeting! Thanks to the effective organization and relaxed scheduling of events, everyone has directly contributed, whether through clear, stimulating invited papers, oral summaries of each poster paper, conversations during extended poster viewing sessions and delicious lunches, or the two scheduled discussion sessions. The latter were remarkably successful under their leaders, Ken Freeman and James Lequeux, who deserve our deep gratitude. The discussions made a conference summary superfluous, but they also provided an excellent lead-in to my highly personal choice of topics mentioned below. In focusing on topics or ideas that caught my attention, I have rarely included attributions to those whose ideas I've borrowed. The often overlapping contributions of participants to the rich fabric woven during IAU 192 suggested this approach, which I hope offends no one.

\section{Sidney's Questions}

The first discussion session got off to a provocative start with four challenging questions posed by Sidney van den Bergh. Here is my brief recapitulation of them and a few of the many remarks that they generated which caught my attention. Note that, as far as I could tell, conclusive answers eluded our collective wisdom!

1. What became in the Local Group of Babul and Ferguson's "boojums", the excess of blue objects seen in the $0.5 \lesssim \mathrm{z} \lesssim 1$ Universe? Where are they, or where is the evidence for significant star bursting 5-7 Gyr ago in the Local Group?

During IAU 192 we were reminded frequently of the ease with which star formation can transform the appearance of galaxies (e.g., the dIr NGC 6822), with the resulting consequence that morphological classification is not necessarily conserved over time. Difficulties of age discrimination on the basis of red giant branches alone factors deeply into our uncertainties concerning star formation histories within the Local Group, too. We were also reminded that the distribution of Milky Way satellites appears to be quite polar. If we started with a spherical distribution, then the implication is that perhaps only $\sim 10 \%$ of the original members have survived intact within the Galactic potential. Thus, the appearance of the Milky Way and of the Local Group as a whole may well have changed over cosmic time in ways difficult to unravel at present. 
2. What is the real luminosity function of faint Local Group members? Can we trust that the inventory is now complete, when the number of dSphs being discovered around M31 is rising as rapidly as demonstrated at this meeting?

In response to this, we heard considerable debate about the traditional visual searches of photographic materials contrasted with what is possible with digitzed sky surveys. The fact that photographic searches found the faint Sextans dSph as a $\sim 6 \sigma$ detection suggest to some that the Milky Way inventory must be nearly complete. However, the angular extent of the Milky Way and its associated nebulosity complicates, or prevents outright, detecting such extremely faint objects over much of the sky. Present-day discovery of faint objects may be primarily in preferential directions, e.g., towards Sculptor, where much research is being conducted and which represents the next major concentration of galaxies beyond the Local Group. Would the digital techniques being used to identify the new M31 dSphs (at $M_{\mathrm{V}} \sim-10$ ) be capable of finding fainter ones, say at -8 ? To what extent could the recent success at finding new dSphs near M31 be due to a higher surface density there? The results from deep, new CCD surveys such as the Sloan Digital Sky Survey may yet provide some surprises for Local Group studies.

3. Do dark matter halos have a minimum mass? Is the observed minimum in the Local Group, $\mathrm{M}_{\text {virial }} \gtrsim 10^{7} \mathrm{M}_{\odot}$, due to a selection effect or to physical processes, such as early mass loss from a system?

Kahn-Woltjer timing arguments set lower limits to the masses of the dark halos for the Milky Way and M31. These masses imply that the dark halos have extents of about $150 \mathrm{kpc}$ if the rotation curves remain flat to large radii, and such sizes should be used when modelling their halos. We were reminded that modern numerical simulations predict a minimum mass to survive supernovae explosions. The well-known example of $\omega$ Cen and its chemical peculiarities suggests a $10^{6} \mathrm{M}_{\odot}$ limit for surviving such explosions. While dSphs look very fragile, with their (apparently) massive dark halos, they are actually small and very dense, and perhaps better likened to cannonballs in the outer halo. Are dark matter halos smooth, as frequently assumed, or are dSphs actually the occasional visible signature of an otherwise quite "blobby" halo?

4. For us, the Local Group plays the role of Noah's Ark, in which we find examples of most types of galaxies seen in the Universe, but why do we not have any of the oversize dSphs that are seen, e.g., in Virgo?

One of these objects is also now known in the M81 group of galaxies. Low surface brightness and angular extent militate against their detection within the Local Group: if one were located at the distance of the Magellanic Clouds, it would be a significant fraction of their angular size. Perhaps digital sky surveys may reveal Local Group analogs of the Virgo objects. However, blind H I surveys are not turning up many good candidates and, when they do, observational confusion with high velocity clouds is proving to be a problem. Another morphological type, gE, missing from the Local Group conceivably could develop should M31 and the Milky Way merge; stay tuned! 


\section{Other Major Themes and Issues of IAU 192}

\subsection{Distances}

At this meeting many new distance determinations have been reported for bona fide and suspected Local Group members, and their subsequent careful analysis may well change our perceptions of the size and shape of the Local Group. Preferences for where to draw the Local Group boundaries (1.2 Mpc? 1.5 Mpc? $1.8 \mathrm{Mpc}$ ? 2.0 Mpc?) were nearly as numerous as participants, and often depended upon how securely the proponent felt the Local Group can be - or should be - distinguished from, e.g., Sculptor.

A topic addressed on day one, but not subsequently revisited, was the cornerstone of the Local Group distance scale, $(m-M)$ for the Magellanic Clouds. This was a major topic at IAU 190 in Victoria, where the vigorous debate was between $\sim 18.50$ and a range of values between 18.60-18.80. While I felt 18.50 emerged the better supported value a few months ago, I was impressed by the increasing clarity of arguments presented here for a higher value, but I doubt that this debate has yet converged.

Similarly, the distance to M31 is debated over a range from $\sim 710$ to $925 \mathrm{kpc}$; what is correct? For dynamical analyses, what is the appropriate velocity of the Andromeda subgroup: should one use the average velocity of all the objects, some $(\cos \theta)^{-1}$ weighting, or ...?

\subsection{Ages of the Oldest Stellar Populations}

From reports here, we seem to have evidence in most Local Group members for a component of very old stellar populations, from the Sagittarius dSph being subsumed into the Milky Way to the objects at (your favorite) edge of the Local Group. To be sure, the fraction of extremely old stars varies considerably from one dSph or galaxy to another, but each seems to have something we might consider a relic of a global original epoch of star formation throughout the Local Group. The massive globular cluster, NGC 2419, in the outer Galactic halo at twice the distance of the Magellanic Clouds, appears to be indistinguishable from M92 in the inner Galactic halo. Similarly, evidence was presented here and in Victoria that globular clusters in the Large Magellanic Cloud - which have disc, rather than halo, kinematices - have the same age as their apparent counterparts in the Milky Way. Are these relative age determinations pointing at a universal "trigger" mechanism or physical process for star and massive star cluster formation, one that operated simultaneously in the proto-Local Group over what is now a large volume?

\subsection{Star Formation Patterns and Histories}

The many new results on stellar populations presented here left me with a sense of wonder at the diversity of Local Group members, especially the less luminous ones! We are at an early stage of quantitatively characterizing that diversity and many questions challenge us. While we have some physical sense of how star formation might be triggered in interacting galaxies, how does star formation ignite in isolated galaxies? How did star formation occur within the last few hundred million years in dSphs where there is little, if any, evidence for significant gas? What causes the apparent physical migration of star formation patterns in 
dSphs like Fornax? What drives the differences seen in star formation efficiency in gas rich systems like M31 and the Milky Way?

\subsection{The Fossil Record: Observed and Synthetic CMDs}

A dominant, exciting theme of IAU 192 has been the explosive growth in systematic exploitation, by many groups, of powerful synthetic color-magnitude diagram (CMD) techniques to interpret photometric observations in terms of star formation histories. The frank discussions of the limitations, whether imposed by the nature of the available observational constraints or by the underlying assumptions of the modelling, have been particularly informative.

Many participants stressed that, in the absence of photometry that reaches the main sequence turnoff(s) in composite stellar systems, the age-metallicity degeneracy for red giants seriously compromises derivation of reliable star formation histories. Examples of quite different insights arising from CMD photometry reaching the main sequence (e.g., Carina, Fornax, Leo I) surfaced repeatedly in this conference. Without that information, the uniqueness of synthetic CMD solutions is difficult, or impossible, to establish. Color information over very wide baselines also exerts important leverage towards establishing unique solutions.

Moreover, differences among theoretical models for red giant branches implant their own strong signatures upon interpretations made with models from one school or another. Maeder provided a particularly sobering analysis regarding current theoretical understanding of massive star evolution. He stressed that models for asymptotic giant branch stars, horizontal branch stars and asymptotic giant branch manqué stars are on questionable grounds, when used for detailed interpretation of observations in terms of absolute luminosities and effective temperatures in synthetic CMD modelling. However, their use for luminosity function predictions, at least in sufficiently coarse boxes, is more likely to yield relatively reliable interpretations of observations at this time.

Another interesting discussion point emerged about current synthetic CMD analyses and the role of binary stars. Some analyses are reporting interpretations from assumed binary fractions as low as $10 \%$, whereas in the solar neighborhood $\sim 60 \%$ is observed. These lead to quantitative differences in the inferred star formation histories, luminosity functions, etc. and need to be treated carefully and parameterized explicitly.

\subsection{Chemistry from Spectroscopy}

The minimal spectroscopic metallicity constraints, to complement the extensive photometric ones for the synthetic CMD interpretations, is perhaps the second most frequently based concern about the elaborate reconstructions of star formation histories in the Local Group presented here. As one participant mused, will the synthetic CMD interpretations "come apart" once spectra are available for the brighter giants? Fortunately, the beautiful observed CMDs presented here are a prerequisite for the multi-object stellar spectroscopy which everyone agrees is vital.

However, how best to attack the much-needed spectroscopic studies remains a concern, as it is not a priori clear what spectral regions and spectral resolution offer the greatest leverage on the underlying astrophysical questions. In the absence of theoretical guidance, observers may follow well trodden paths where we 
know results can be obtained. One such is the infrared calcium triplet, which is a powerful and convenient probe for intrinsically luminous red giants throughout the Local Group. With 4-m class telescopes the triplet is often by far the most accessible diagnostic for distant, faint stars. However, recent studies have raised concerns about the reliability of an $\alpha$ element as a surrogate for $[\mathrm{Fe} / \mathrm{H}]$. The widely observed rise from solar ratios in the Galactic disk to $[\alpha / \mathrm{Fe}] \sim+0.4$ in the Galactic halo field star population is not universal throughout the halo, apparently in reflection of differing histories of supernova contributions in different regions. Star formation histories derived from equating triplet metallicties with $[\mathrm{Fe} / \mathrm{H}]$ will be subject to the additional, potentially significant parameter $[\alpha / \mathrm{Fe}]$ when the latter is unknown. Perhaps a more fundamental question to ask stellar modellers is, what is the most important chemical information needed to secure unique, reliable answers in our quest to interpret CMDs in terms of Local Group evolution?

More generally, there has been little mention at IAU 192 of the long standing second-parameter issue, which suggests that many now appear to believe is a solved problem, given the well argued case from synthetic horizontal branch models for differences in age being the dominant cause of the effect among Galactic globular clusters and dSphs. Yet some caution that the role of chemistry or other physical effects (e.g., mass loss during ascent of the red giant branch by low-mass stars, internal mixing driven by stellar angular momentum, etc.) may be equally important. While the total number of horizontal branch stars in a population may be reasonably well predicted, their distribution remains uncertain. Reconstructions of star formation histories and the assembling of galaxies within the Local Group will be influenced by the ultimate resolution to this puzzle.

\subsection{Chemical Evolution Within The Local Group}

Some participants raised concern about the apparent composition differences between the interstellar gas and the young stars born therefrom, as evidenced in the Magellanic Clouds, for instance. Others worry deeply whether chemical evolution in Local Group objects can be satisfactorily described by a closed-box approximation, or if significant amounts of gas inflow or outflow will be required.

From a theoretical stellar evolutionary viewpoint, we heard that the role of rotationally driven mixing in massive stars may be much greater than heretofore believed and that it may be highly dependent upon metallicity. Therefore, the apparent distribution of chemical elements may be more a product of such rotationally driven stellar mixing cycles than generally appreciated. The intriguing suggestion was made that perhaps stellar axial rotation rates are different from galaxy to galaxy. If any of the above were true, we would be making erroneous interpretations based upon the available, limited chemical and photometric information from the intrinsically luminous stars in reach of our telescopes. We are thus challenged to learn how to identify and deal with such biases when interpreting Local Group history.

Moving towards a more global context, I continue to find it fascinating that we could go outside this evening with an ordinary pair of binoculars to view metal-deficient objects within the Local Group, whose chemistry overlaps 
that of high redshift quasar absorption line systems. The high redshift Universe appears to be remarkably near at hand, which brings me to my final two remarks.

\section{A Common Misconception and An Indisputable Fact}

\subsection{A Common Misconception}

There were so many presentations with similar themes based upon the powerful synthetic CMD technique at the meeting, that, by the time Antonio Aparicio was to give his, he instead discussed a series of common misconceptions about the approach and its results. His final, tongue-in-cheek one resonates with me as I review the many fundamental issues raised during IAU 192: "Cosmology is important; the Local Group is not." The inseparability of the Local Group from most of the major problems of star and galaxy formation and cosmology has permeated this meeting, whose ideas and discussions will influence our research for many years to come.

\subsection{An Indisputable Fact}

We have experienced many controversies and strong differences of opinion or interpretation during this excellent meeting, which is exactly as it should be in a subject as vital as that of "The Stellar Content of the Local Group." There is, however, one thing upon which all will agree: we offer our profound thanks and congratulations to the Scientific and Local Organizing Committees. This first International Astronomical Union Symposium in Africa has been $\mathbf{1 0 0 \%}$ successful scientifically, and I am certain that many more IAU sponsored meetings will deservedly follow. To our hosts, I offer special thanks from all of us for your outstanding hospitality, which has left so many wonderful memories for each of us to savor for the remainder of our lives. We also extend best wishes to you for the speedy implementation of the South African Large Telescope, which promises solutions to so many of the challenges raised by our discussions in Cape Town during the past week. 